\title{
Symptomatische KHK-Behandlung im Praxisalltag
}

\section{Exklusive Herzfrequenzreduktion holt die Lebensqualität zurück}

- Patienten mit stabiler Angina pectoris leben wieder auf, wenn sie zusätzlich zur Betablocker-Standardtherapie den $I_{\mathrm{f}^{-}} \mathrm{Ka}$ nal-Hemmer Ivabradin (Procoralan ${ }^{\circledR}$, Fa. Servier) erhalten, da die Zusatztherapie Angina-pectoris-Beschwerden effektiv reduziert. „Dies bedeutet für die Patienten eine deutliche Verbesserung ihrer Lebensqualität, denn sie können wieder aktiver am Leben teilnehmen", betonte Priv.-Doz. Dr. Henning Ebelt, Halle.

Ivabradin hemmt selektiv den "Schrittmacher-Strom " $I_{\mathrm{f}}$ in den Schrittmacherzellen des Sinusknotens und reduziert so exklusiv die Herzfrequenz. Andere kardiale Funktionen werden nicht beeinflusst. Klinische Studien belegten, dass die unter einer Betablockertherapie eingeschränkte Belastbarkeit unter Ivabradin wieder hergestellt wird.

Die in Deutschland durchgeführte, nicht interventionelle Studie ADDITONS belegte erstmals den Nutzen der exklusiven Herzfrequenzreduktion mit Ivabradin in der
Abbildung 1

Angina-pectoris-Beschwerden

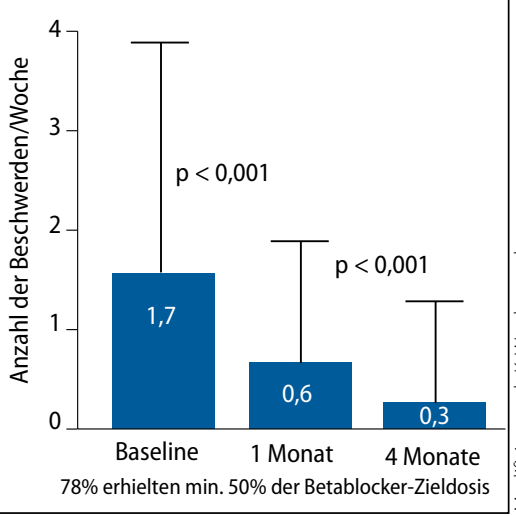

Abb. 1 Effektive Reduktion von Anginapectoris-Beschwerden unter der Kombination mit Ivabradin.

Kombination mit verschiedenen Betablockern im Hinblick auf die Symptomatik und Lebensqualität von KHK-Patienten mit stabiler Angina pectoris unter Alltagsbedingungen. Nach vier Monaten Therapie wurden die Daten von 2330 Patienten ausge- wertet. 57\% der Patienten hatten bereits eine Angioplastie oder Bypass-Operation erhalten und bei $36 \%$ war anamnestisch ein Myokardinfarkt bekannt. Alle Patienten erhielten Betablocker (Metoprolol 43\%, Bisoprolol 37\%, Nebivolol 13\%, Carvedilol 7\%).

Nach vier Monaten Ivabradin hatte die mittlere Herzfrequenz um 19,4 Schläge auf 65,6 Schläge/min abgenommen. Dies ging mit einer signifikanten Abnahme von Angina-pectoris-Beschwerden einher (Abb. 1). Vor Studienbeginn war mehr als die Hälfte der Patienten (51\%) nach den Kriterien der Canadian Cardiovascular Society (CCS) mit einem Angina-pectoris-Schweregrad CCS II eingestuft worden. Nach vier Monaten wies der Großteil (68\%) einen CCS I auf. Entsprechend nahm auch der Nitratverbrauch ab. Gleichzeitig verbesserte sich die Belastbarkeit. Dies ging mit einer signifikanten Verbesserung der Lebensqualität einher.

- Dr. med. Kirsten Westphal

Quelle: Posterpräsentation im Rahmen des ESC-Kongresses, Paris, August 2011

\section{Diabetische Neuropathie}

\section{Pregabalin moduliert überaktivierte Neuronen}

- Die wichtigste kausale Therapie, um eine diabetischen Neuropathie zu verhindern, ist die optimale Diabeteseinstellung. „Je besser die Stoffwechselsituation, umso geringer ist das Risiko für eine solche Folgeschädigung", sagte Prof. Jochen Seufert, Freiburg.

Bei vielen Betroffenen führt die diabetische Neuropathie zu Schmerzen und unangenehmen Missempfindungen. Dann ist eine zusätzliche Behandlung erforderlich. "Wichtig ist es, den Schmerz rasch und wirkungsvoll zu behandeln, damit er sich nicht im Schmerzgedächtnis festsetzt", so Seufert. In erster Linie kommen Antidepressiva wie Duloxetin oder Pregabalin in Betracht. Diese Substanzen sind gemäß der Praxisleitlinie der Deutschen DiabetesGesellschaft als Medikamente der ersten Wahl anzusehen.
Pregabalin (Lyrica®) ist eine Aminosäure, die leicht die Blut-Hirn-Schranke passiert. Ihre günstige klinische Wirkung entfaltet sie über eine Modulation der bei der diabetischen Neuropathie überaktivierten Neurone, d. h. die Freisetzung exzitatorischer Neurotransmitter wird inhibiert.

Die überzeugende Wirksamkeit konnte in acht doppelblinden Studien nachgewiesen werden mit Dosierungen zwischen 150 und $600 \mathrm{mg}$ täglich. „Dadurch kam es zu einer signifikanten Schmerzreduktion bereits innerhalb einer Woche und anhaltend über den gesamten Behandlungszeitraum", so Seufert. In einer entsprechenden Metaanalyse konnte bei fast $50 \%$ der mit Pregabalin Behandelten eine mindestens 50\%ige Abnahme des Schmerzscores dokumentiert werden. Die Patienten berichteten auch über eine deutliche Verbesserung ihres All- gemeinzustandes und Abnahme der schmerzbedingten Schlafstörungen.

Die Therapie sollte mit $150 \mathrm{mg}$ täglich begonnen und je nach Ansprechen und Verträglichkeit nach drei bis sieben Tagen auf 300 mg täglich erhöht werden. „Bei Bedarf ist eine weitere Steigerung nach sieben Tagen auf eine Höchstdosis von 600 mg täglich sinnvoll“, so Seufert. Das Medikament sollte unabhängig von den Mahlzeiten eingenommen und die Dosis bei eingeschränkter Nierenfunktion reduziert werden. Daneben empfiehlt sich die Anwendung physiotherapeutischer Maßnahmen wie z. B. die elektrische Nerven- oder Muskelstimulation.

\footnotetext{
Dr. med. Peter Stiefelhagen

Quelle: Satellitensymposium „Der Patient mit

Diabetes von A wie Auge bis Z wie Zehe", DDG-

Kongress, Leipzig, Juni 2011 (Veranstalter: Pfizer)
} 